\title{
Diffusive cosmic ray acceleration at shock waves of arbitrary speed
}

\author{
R. Schlickeiser*t \\ Institut für Theoretische Physik, Lehrstuhl IV: Weltraum- und Astrophysik, Ruhr-Universität \\ Bochum, D-44780 Bochum, Germany \\ E-mail: rschetp4.rub.de
}

The analytical theory of diffusive acceleration of cosmic rays at parallel stationary shock waves of arbitrary speed with magnetostatic turbulence is developed from first principles. The theory is based on the diffusion approximation to the gyrotropic cosmic ray particle phase space distribution functions in the respective rest frames of the up- and downstream medium. We derive the correct cosmic ray jump conditions for the cosmic ray current and density, and match the up- and downstream distribution functions at the position of the shock. It is essential to account for the different particle momentum coordinates in the up- and downstream media. Analytical expressions for the momentum spectra of shock-accelerated cosmic rays are calculated. These are valid for arbitrary shock speeds including relativistic shocks. The correctly taken limit for nonrelativistic shock speeds leads at relativistic cosmic-ray momenta to the power-law momentum spectrum $F_{1}\left(y_{1}\right) \propto y_{1}^{-q(r)}$ and at nonrelativistic cosmic-ray momenta to the power-law momentum spectrum $F_{1}\left(y_{1}\right) \propto y_{1}^{-(1+q(r))}$, where the power-law spectral index $q(r)$ is a factor 2 greater than the standard spectral index from nonrelativistic shock acceleration theory. Moreover, for nonrelativistic shock speeds we calculate for the first time the injection velocity threshold $\beta_{c} \geq \sqrt{3} \beta_{u}$, settling the long-standing injection problem for nonrelativistic shock acceleration.

35th International Cosmic Ray Conference - ICRC2017

10-20 July, 2017

Bexco, Busan, Korea

\footnotetext{
* Speaker.

$\dagger$ This work was partially supported by the Deutsche Forschungsgemeinschaft through grants Schl 201/29-1 and Schl 201/34-1.
} 


\section{Introduction}

Apart from magnetic reconnection in transient electric fields cosmic-ray particles in magnetized cosmic plasmas are accelerated by first- and second-order Fermi processes interacting with low-frequency electromagnetic fluctuations such as Alfven and Whistler plasma waves. First-order Fermi acceleration at shock waves is a prime candidate for particle acceleration in astrophysics process because of the large kinetic energy reservoir of supersonic outflows. Pulsar wind nebulae, active galactic nuclei and gamma-ray bursts exhibit highly collimated winds or jets with initial relativistic bulk Lorentz factors $\Gamma_{0}=\left(1-\left(V_{0} / c\right)^{2}\right)^{-1 / 2}$ up to $100-10^{3}$. Therefore there is high interest to understand the acceleration of cosmic rays at magnetized shocks of arbitrary speed.

\section{First principles}

To keep the mathematical complexities at a minimum, we consider the case of an infinitely extended planar shock with a step-like shock profile $U\left(z^{*}<0\right)=\beta_{u} c, U\left(z^{*}>0\right)=\beta_{d} c$ with positive $0<\beta_{d}<\beta_{u}$. In order to automatically remove the strong particle momentum anisotropy due to the relativistically moving medium, we take the cosmic-ray phase space coordinates in the mixed comoving coordinate system: time $t^{*}$ and space coordinates $z^{*}$ in the laboratory (=shock rest) system and particle's momentum coordinates $p$ and $\mu=p_{\|} / p$ in the rest frame of the streaming plasma. This choice then allows us to apply the diffusion approximation to cosmic-ray transport in the respective up- and downstream media. Let $(p, \mu)$ and $f$ represent either $\left(p_{1}, \mu_{1}\right)$ and $f_{u}$ or $\left(p_{2}, \mu_{2}\right)$ and $f_{d}$, respectively. It is also useful to keep the parallel momentum coordinate $p_{\|}=p \mu$. It is essential that, because of the chosen mixed comoving coordinate system, the up- $\left(p_{1}, \mu_{1}\right)$ and downstream $\left(p_{2}, \mu_{2}\right)$ cosmic-ray particle momenta in general are different.

In both reference systems the Larmor-phase averaged steady-state Fokker-Planck transport equation (without momentum losses) for the anisotropic but gyrotropic cosmic-ray phase space density $f\left(z^{*}, p, \mu\right)$ in a medium with magnetostatic turbulence, propagating with the stationary bulk speed $\vec{U}=U(z) \overrightarrow{z_{z^{*}}}=\beta_{s} c \overrightarrow{e_{z^{*}}}$ with $\Gamma=\left[1-\beta_{s}^{2}\right]^{-1 / 2}$ aligned along the magnetic field direction, reads (Lindquist 1966; Kirk, Schneider and Schlickeiser 1988)

$$
\Gamma[U+v \mu]\left[\frac{\partial f_{0}}{\partial z^{*}}-\frac{d U}{d z^{*}} \Gamma^{2} \frac{p}{v} \frac{\partial f_{0}}{\partial p_{\|}}\right]=Q\left(z^{*}, p, \mu\right)+\frac{\partial}{\partial \mu}\left[D_{\mu \mu}(\mu) \frac{\partial f_{0}}{\partial \mu}\right],
$$

where $Q\left(z^{*}, p_{1}, \mu_{1}\right)=Q_{0}\left(p_{1}, \mu_{1}\right) \delta\left(z^{*}\right)$ is the assumed particle injection rate solely at the position of the shock. With $d(\Gamma U) / d z^{*}=\Gamma^{3}\left(d U / d z^{*}\right)$ the transport equation (2.1) can be written as

$$
\Gamma[U+v \mu] \frac{\partial f_{0}}{\partial z^{*}}-\frac{d(U \Gamma)}{d z^{*}}(U+v \mu) \frac{p}{v} \frac{\partial f_{0}}{\partial p_{\|}}=Q_{0}\left(p_{1}, \mu_{1}\right) \delta\left(z^{*}\right)+\frac{\partial}{\partial \mu}\left[D_{\mu \mu}(\mu) \frac{\partial f_{0}}{\partial \mu}\right]
$$

With dimensionless momentum coordinates $y=p /(m c), y_{\|}=p_{\|} /(m c)$ Eq. (2.2) after a few manipulations reads

$$
\frac{\partial S\left(z^{*}, y, y_{\|}\right)}{\partial z^{*}}+\Gamma \beta_{s} c \frac{\partial}{\partial z^{*}} \frac{\partial}{\partial y_{\|}}\left[\left(\beta_{s} \sqrt{1+y^{2}}+y_{\|}\right) f_{0}\right]=Q_{0}\left(y_{1}, \mu_{1}\right) \delta\left(z^{*}\right)+\frac{\partial}{\partial \mu}\left[D_{\mu \mu}(\mu) \frac{\partial f_{0}}{\partial \mu}\right]
$$


with the cosmic-ray current

$$
S\left(z^{*}, y, y_{\|}\right)=\frac{c y_{\|} f_{0}}{\Gamma \sqrt{1+y^{2}}}-c \beta_{s} \Gamma\left(\beta_{s} \sqrt{1+y^{2}}+y_{\|}\right) \frac{\partial f_{0}}{\partial y_{\|}}
$$

Eq. (2.3) represents the generalization of the transport equation of Gleeson and Axford (1967) accounting for shocks of arbitrary speed and anisotropic but gyrotropic cosmic-ray particle distribution functions. For nonrelativistic shocks Gleeson and Axford (1967) showed that, apart from sources, the cosmic-ray "jump" conditions at a shock front are simply that the isotropic phase space density and the isotropic current $F\left(y, z^{*}\right)=\int_{-1}^{1} d \mu f_{0}\left(y, \mu, z^{*}\right) / 2, \bar{S}\left(y, z^{*}\right)=\int_{-1}^{1} d \mu S\left(y, \mu, z^{*}\right) / 2$ are continuous.

\subsection{Diffusion approximation in the up- and down-stream medium}

For the step-like shock velocity profile the Fokker-Planck transport equation (2.2) in the up$\left(z^{*}<0\right)$ and downstream $\left(z^{*}>0\right)$ medium is given by

$$
\frac{\partial}{\partial z^{*}}\left[\Gamma(U+v \mu) f_{0}\right]=\frac{\partial}{\partial \mu}\left[D_{\mu \mu}(\mu) \frac{\partial f_{0}}{\partial \mu}\right],
$$

We write $f_{0}\left(z^{*}, y, \mu\right)=F\left(z^{*}, y\right)+g\left(z^{*}, y, \mu\right)$ as the sum of the isotropic part of the cosmic-ray phase space density $F\left(z^{*}, y\right)$ and the anisotropy $g\left(z^{*}, y, \mu\right)$. For small anisotropies $\left|g\left(z^{*}, y, \mu\right)\right| \ll F\left(z^{*}, p\right)$ the diffusion approximation (Jokipii 1966) provides the diffusion-convection transport equation for the isotropic phase space density $F\left(z^{*}, p\right)$ and the anisotropy $g\left(z^{*}, y, \mu\right)$

$$
\begin{gathered}
\frac{\partial}{\partial z} \Gamma\left[U F-\Gamma \kappa \frac{\partial F}{\partial z^{*}}\right]=0, g\left(z^{*}, y, \mu\right)=\frac{v \Gamma}{4} A(\mu) \frac{\partial F\left(z^{*}, y\right)}{\partial z^{*}}, A(\mu)=\int_{-1}^{1} d \mu \frac{(1-\mu)\left(1-\mu^{2}\right)}{D_{\mu \mu}(\mu)} \\
-2 \int_{-1}^{\mu} d x \frac{\left(1-x^{2}\right)}{D_{\mu \mu}(x)}, \kappa=-\frac{v^{2}}{8} \int_{-1}^{1} d \mu \mu A(\mu)=\frac{v^{2}}{8} \int_{-1}^{1} d \mu \frac{\left(1-\mu^{2}\right)^{2}}{D_{\mu \mu}(\mu)}
\end{gathered}
$$

Demanding as spatial boundary conditions $F_{u}\left(z^{*}=-\infty, y_{1}\right)=0, F_{d}\left(z^{*}=\infty, y_{2}\right)=F_{2}\left(y_{2}\right)$, finite $F_{u}\left(z^{*}=0, y_{1}\right)$ and $F_{d}\left(z^{*}=0, y_{2}\right)$ at the shock location $z^{*}=0$, and spatially-independent diffusion coefficients $\kappa_{1,2}$, the approximated up- and downstram anisotropic (but gyrotropic) phase space distribution functions are

$$
\begin{aligned}
f_{u}\left(z^{*} \leq 0, y_{1}, \mu_{1}\right) & \simeq f_{u}\left(0, y_{1}, \mu_{1}\right) e^{\frac{\beta_{u} c^{*} z^{*}}{\Gamma_{u} k_{1}}}, f_{u}\left(0, y_{1}, \mu_{1}\right)=F_{1}\left(y_{1}\right) B\left(\mu_{1}\right), f_{d}\left(z^{*} \geq 0, y_{2}, \mu_{2}\right)=f_{u}\left(0, y_{1}, \mu_{1}\right) \\
& \simeq F_{2}\left(y_{2}\right), B\left(\mu_{1}\right)=1+\frac{\beta_{u} \beta_{1} c^{2}}{4 \kappa_{1}} A\left(\mu_{1}\right)=1-\frac{2 \beta_{u}}{\beta_{1}} \frac{A\left(\mu_{1}\right)}{\int_{-1}^{1} d \mu_{1} \mu_{1} A\left(\mu_{1}\right)}
\end{aligned}
$$

Mostly important is the isotropy of the downstream distribution function in its rest frame!

\subsection{Momentum spectrum of accelerated particles at the shock}

We determine the momentum spectrum of accelerated cosmic rays at the shock by integrating Eq. (2.3) from $z^{*}=-\eta$ to $z^{*}=\eta$ and considering the limit $\eta \rightarrow 0$ resulting in

$$
S_{d}\left(0, y_{2}, y_{\|, 2}\right)-S_{u}\left(0, y_{1}, y_{\|, 1}\right)-Q_{0}\left(y_{1}, \mu_{1}\right)
$$




$$
\begin{gathered}
+c \Gamma_{d} \beta_{d} \lim _{\eta \rightarrow 0} \frac{\partial}{\partial y_{\|, 2}}\left[\left(\beta_{d} \sqrt{1+y_{2}^{2}}+y_{\|, 2}\right)\right]\left[f_{d}\left(\eta, y_{2}, y_{\|, 2}\right)-f_{d}\left(0, y_{2}, y_{\|, 2}\right)\right] \\
+c \Gamma_{u} \beta_{u} \lim _{\eta \rightarrow 0} \frac{\partial}{\partial y_{\|, 1}}\left[\left(\beta_{u} \sqrt{1+y_{1}^{2}}+y_{\|, 1}\right)\right]\left[f_{u}\left(0, y_{1}, y_{\|, 1}\right)-f_{u}\left(-\eta, y_{1}, y_{\|, 1}\right)\right] \\
=\lim _{\eta \rightarrow 0}\left[\int_{-\eta}^{0} d z^{*} \frac{\partial}{\partial \mu_{1}} D_{\mu \mu}\left(\mu_{1}\right) \frac{\partial f_{u}\left(z^{*}, y_{1}, \mu_{1}\right)}{\partial \mu_{1}}+\int_{0}^{\eta} d z^{*} \frac{\partial}{\partial \mu_{2}} D_{\mu \mu}\left(\mu_{2}\right) \frac{\partial f_{d}\left(z^{*}, y_{2}, \mu_{2}\right)}{\partial \mu_{2}}\right]
\end{gathered}
$$

With the solutions (2.7) from the diffusion approximation the majority of terms in this lengthly equation vanishes, leaving only

$$
S_{d}\left(0, y_{2}, y_{\|, 2}\right)-S_{u}\left(0, y_{1}, y_{\|, 1}\right)=Q_{0}\left(y_{1}, \mu_{1}\right)
$$

with

$$
\begin{gathered}
S_{d}\left(0, y_{2}, y_{\|, 2}\right)=\frac{c y_{\|, 2} f_{d}\left(0, y_{2}, y_{\|, 2}\right)}{\Gamma_{d} \sqrt{1+y_{2}^{2}}}-c \beta_{d} \Gamma_{d}\left(\beta_{d} \sqrt{1+y_{2}^{2}}+y_{\|, 2}\right) \frac{\partial f_{d}\left(0, y_{2}, y_{\|, 2}\right)}{\partial y_{\|, 2}}, \\
S_{u}\left(0, y_{1}, y_{\|, 1}\right)=\frac{c y_{\|, 1} f_{u}\left(0, y_{2}, y_{\|, 1}\right)}{\Gamma_{u} \sqrt{1+y_{1}^{2}}}-c \beta_{u} \Gamma_{u}\left(\beta_{u} \sqrt{1+y_{1}^{2}}+y_{\|, 1}\right) \frac{\partial f_{u}\left(0, y_{1}, y_{\|, 1}\right)}{\partial y_{\|, 1}}
\end{gathered}
$$

Together with the continuity condition (Kirk and Schneider 1987)

$$
f_{u}\left(z^{*}=0, y_{1}, \mu_{1}\right)=f_{d}\left(z^{*}=0, y_{2}, \mu_{2}\right)
$$

Eqs. (2.9) - (2.11) determine the momentum spectrum of accelerated particles at the shock. However, these equations still contain $\left(y_{\|, 1}, y_{1}\right)$ and $\left(y_{\|, 2}, y_{2}\right)$ which are related to each other using relativistic kinematics. If

$$
b=\frac{\beta_{u}-\beta_{d}}{1-\beta_{u} \beta_{d}}=\frac{\beta_{u}(r-1)}{r-\beta_{u}^{2}}, \Gamma_{r}=\left(1-b^{2}\right)^{-1 / 2}=\Gamma_{u} \Gamma_{d}\left(1-\beta_{u} \beta_{d}\right)
$$

denote the relative velocity of the upstream medium with respect to the downstream medium and the associated relative Lorentz factor, one finds generally with $r=\beta_{u} / \beta_{d}$ and $\beta_{1}=y_{1} / \sqrt{1+y_{1}^{2}}$

$$
\begin{gathered}
y_{2}=\sqrt{\Gamma_{r}^{2}\left(b \mu_{1} y_{1}+\sqrt{1+y_{1}^{2}}\right)^{2}-1}, \mu_{2}=\frac{\Gamma_{r}\left(y_{1} \mu_{1}+b \sqrt{1+y_{1}^{2}}\right)}{\sqrt{\Gamma_{r}^{2}\left(b \mu_{1} y_{1}+\sqrt{1+y_{1}^{2}}\right)^{2}-1}}, \\
\frac{\partial}{\partial y_{2}}=\frac{1}{\left(1+b \beta_{1} \mu_{1}\right) \sqrt{\Gamma_{r}^{2}\left(b \mu_{1} y_{1}+\sqrt{1+y_{1}^{2}}\right)^{2}-1}}\left[\left(\beta_{1}+b \mu_{1}\right) \sqrt{1+y_{1}^{2}} \frac{\partial}{\partial y_{1}}+\frac{b\left(1-\mu_{1}^{2}\right)}{y_{1} \sqrt{1+y_{1}^{2}}} \frac{\partial}{\partial \mu_{1}}\right] \\
\frac{\partial \mu_{2}}{\partial \mu_{1}}=\frac{\Gamma_{r} y_{1}\left(1+\frac{b \mu_{1}}{\beta_{1}}\right)}{\left[\Gamma_{r}^{2}\left(b \mu_{1} y_{1}+\sqrt{1+y_{1}^{2}}\right)^{2}-1\right]^{3 / 2}}
\end{gathered}
$$




\section{Transport equation at the shock}

In terms of spherical momentum coordinates the up- and downstream cosmic-ray currents (2.10) at the shock are

$$
\begin{gathered}
\frac{S_{u}\left(0, y_{1}, \mu_{1}\right)}{c \beta_{u} \Gamma_{u}}=\left[1+\frac{\beta_{1} \mu_{1}}{\beta_{u}}+\right. \\
\left.2 \mu_{1}\left(\mu_{1}+\frac{\beta_{u}}{\beta_{1}}\right)\right] F_{1}\left(y_{1}\right) B\left(\mu_{1}\right)-F_{1}\left(y_{1}\right)\left(1-\mu_{1}^{2}\right) \frac{\partial}{\partial \mu_{1}}\left[\left(\mu_{1}+\frac{\beta_{u}}{\beta_{1}}\right) B\left(\mu_{1}\right)\right] \\
-\frac{1}{y_{1}^{2}} \frac{\partial}{\partial y_{1}}\left[y_{1}^{3} F_{1}\left(y_{1}\right) \mu_{1}\left(\mu_{1}+\frac{\beta_{u}}{\beta_{1}}\right) B\left(\mu_{1}\right)\right]
\end{gathered}
$$

and, as $f_{d}\left(0, y_{2}, \mu_{2}\right)=F_{2}\left(y_{2}\right)=F_{1}\left(y_{1}\right) I\left(b, y_{1}\right)$ is independent of $\mu_{2}$,

$$
\frac{S_{d}\left(0, y_{2}, \mu_{2}\right)}{c \beta_{d} \Gamma_{d}}=\mu_{2}\left[\mu_{2}+\frac{\beta_{2}}{\beta_{d}}+2\left(\mu_{2}+\frac{\beta_{d}}{\beta_{2}}\right)\right] F_{1}\left(y_{1}\right) I\left(b, y_{1}\right)-\frac{1}{y_{2}^{2}} \frac{\partial}{\partial y_{2}}\left[y_{2}^{3} \mu_{2}\left(\mu_{2}+\frac{\beta_{d}}{\beta_{2}}\right) F_{1}\left(y_{1}\right) I\left(b, y_{1}\right)\right]
$$

where we use the continuity condition (2.11) and the last equation (2.13) to relate

$$
\begin{aligned}
F_{2}\left(y_{2}\right) & =\frac{1}{2} \int_{-1}^{1} d \mu_{2} f_{d}\left(0, y_{2}, \mu_{2}\right)=\frac{1}{2} \int_{-1}^{1} d \mu_{2} f_{u}\left(0, y_{1}, \mu_{1}\right)=\frac{F_{1}\left(y_{1}\right)}{2} \int_{-1}^{1} d \mu_{1} \frac{\partial \mu_{2}}{\partial \mu_{1}} B\left(\mu_{1}\right) \\
= & I\left(b, y_{1}\right) F_{1}\left(y_{1}\right), I\left(b, y_{1}\right)=\frac{\Gamma_{r} y_{1}}{2} \int_{-1}^{1} d \mu_{1} \frac{B\left(\mu_{1}\right)\left(1+\frac{b \mu_{1}}{\beta_{1}}\right)}{\left[\Gamma_{r}^{2}\left(b \mu_{1} y_{1}+\sqrt{1+y_{1}^{2}}\right)^{2}-1\right]^{3 / 2}}
\end{aligned}
$$

With Eqs. (2.13) we express the downstream current (3.2) solely in terms of upstream momenta. The cosmic-ray jump condition (2.9) then yields

$$
\begin{gathered}
\beta_{d} \Gamma_{d} F_{1}\left(y_{1}\right) I\left(b, y_{1}\right)\left(b+\beta_{1} \mu_{1}\right)\left(\frac{1}{\beta_{d}\left(1+b \beta_{1} \mu_{1}\right)}+\frac{b+\beta_{1} \mu_{1}}{\beta_{1}^{2} W\left(b, \mu_{1}, y_{1}\right)}+\frac{2\left[\beta_{d}\left(1+b \beta_{1} \mu_{1}\right)+b+\beta_{1} \mu_{1}\right]}{\beta_{1}^{2} W\left(b, \mu_{1}, y_{1}\right)}\right) \\
-\frac{\beta_{d} \Gamma_{d}}{\left(1+b \beta_{1} \mu_{1}\right) y_{1}^{3} W^{\frac{3}{2}}\left(b, \mu_{1}, y_{1}\right)}\left(\left(\beta_{1}+b \mu_{1}\right) \sqrt{1+y_{1}^{2}} \frac{\partial}{\partial y_{1}}+\frac{b\left(1-\mu_{1}^{2}\right)}{y_{1} \sqrt{1+y_{1}^{2}}} \frac{\partial}{\partial \mu_{1}}\right) \\
\left(\left(1+y_{1}^{2}\right) y_{1}\left(b+\beta_{1} \mu_{1}\right) W^{1 / 2}\left(b, \mu_{1}, y_{1}\right)\left[b+\beta_{1} \mu_{1}+\beta_{d}\left(1+b \beta_{1} \mu_{1}\right)\right] F_{1}\left(y_{1}\right) I\left(b, y_{1}\right)\right) \\
-\left[1+\frac{\beta_{1} \mu_{1}}{\beta_{u}}+2 \mu_{1}\left(\mu_{1}+\frac{\beta_{u}}{\beta_{1}}\right)\right] \beta_{u} \Gamma_{u} F_{1}\left(y_{1}\right) B\left(\mu_{1}\right)+\beta_{u} \Gamma_{u} F_{1}\left(y_{1}\right)\left(1-\mu_{1}^{2}\right) \frac{\partial}{\partial \mu_{1}}\left[\left(\mu_{1}+\frac{\beta_{u}}{\beta_{1}}\right) B\left(\mu_{1}\right)\right] \\
+\frac{\beta_{u} \Gamma_{u}}{y_{1}^{2}} \frac{\partial}{\partial y_{1}}\left[y_{1}^{3} F_{1}\left(y_{1}\right) \mu_{1}\left(\mu_{1}+\frac{\beta_{u}}{\beta_{1}}\right) B\left(\mu_{1}\right)\right]=\frac{Q_{0}\left(y_{1}, \mu_{1}\right)}{c}
\end{gathered}
$$

with $W\left(b, \mu_{1}, y_{1}\right)=\left(1+\left(b \mu_{1} / \beta_{1}\right)\right)^{2}+\left(b^{2}\left(1-\mu_{1}^{2}\right) / y_{1}^{2}\right)$. This equation holds for cosmic-ray particles of arbitrary momentum, shock waves of arbitrary speed and and general injection functions $Q_{0}\left(y_{1}, \mu_{1}\right)$.

As we are particularly interested in the isotropic momentum spectrum $F_{1}\left(y_{1}\right)$ of accelerated particles at the shock. we average Eq. (3.4) over $\mu_{1}$ leading after lengthly algebra (Schlickeiser and Oppotsch 2017) to

$$
\Omega\left(b, y_{1}\right) F_{1}\left(y_{1}\right)+\frac{1}{y_{1}^{2}} \frac{d}{d y_{1}}\left(y_{1}^{3} F_{1}\left(y_{1}\right) T\left(b, y_{1}\right)\right)=\frac{\int_{-1}^{1} d \mu_{1} Q_{0}\left(y_{1}, \mu_{1}\right)}{2},
$$

in terms of the convection rate $\Omega\left(b, y_{1}\right)$ and the acceleration rate $T\left(b, y_{1}\right)$. 


\subsection{Solution of the transport equation at the shock}

For the isotropic monomomentum injection rate $Q_{0}\left(y_{1}, \mu_{1}\right)=Q_{1} \delta\left(y_{1}-y_{0}\right)$ the solution of Eq. (3.5) is given by

$$
F_{1}\left(y_{1} \geq y_{0}\right)=\frac{3 Q_{1} y_{0}^{2}}{y_{1}^{3} T\left(y_{1}, b\right)} \exp \left[-\int_{y_{0}}^{y_{1}} d y \frac{\Omega(b, y)}{y T(b, y)}\right]
$$

For the (in general momentum-dependent) power-law spectral index we obtain

$$
q\left(r, y_{1}\right)=-\frac{d\left(\ln F_{1} / d\left(y / y_{0}\right)\right)}{d \ln \left(y_{1} / y_{0}\right)}=3+y_{1} \frac{d \ln T\left(b, y_{1}\right)}{d y_{1}}+\frac{\Omega\left(b, y_{1}\right)}{T\left(b, y_{1}\right)},
$$

depending on the ratio of the convection rate $\Omega\left(y_{1}, b\right)$ and the acceleration rate $T\left(y_{1}, b\right)$.

\subsection{Extreme nonrelativistic shock speeds}

In the limit of extremely nonrelativistic shock speeds with $b=0$ and $\Gamma_{r}=1$ we find $W(b=$ $\left.0, \mu_{1}, y_{1}\right)=1$ and $I\left(b=0, y_{1}\right)=1$, so that the transport equation (3.5) for relativistic cosmic rays reduces to

$$
\beta_{d} F_{1}\left(y_{1}\right)+\frac{\beta_{u}-\beta_{d}}{3 y_{1}^{2}} \frac{d\left(y_{1}^{3} F_{1}\left(y_{1}\right)\right)}{d y_{1}}=\frac{1}{2 c} \int_{-1}^{1} d \mu_{1} Q_{0}\left(y_{1}, \mu_{1}\right),
$$

agreeing exactly with the transport equation used in nonrelativistic acceleration theory. However, this case is somewhat unphysical as $b \simeq \beta_{u}-\beta_{d}=0$ implies $\beta_{u}=\beta_{d}$, so that no nonrelativistic shock occurs.

\subsection{Correct limit of nonrelativistic shocks}

The correct limit of nonrelativistic shocks with small but finite values of $b=(r-1) \beta_{d} \ll 1$ yields to second order in $\beta_{d} \ll 1$ for the convection and acceleration rates

$$
\begin{gathered}
\Omega\left(b \ll 1, y_{1}\right) \simeq c \beta_{d}\left[r-\frac{r-1}{3} \beta_{1}^{2}+\frac{\beta_{d}^{2} r(r-1)(7 r-1)}{3 \beta_{1}^{2}}\right], \\
T\left(b \ll 1, y_{1}\right) \simeq \frac{c \beta_{d} r}{3}\left(1-3 \frac{r^{2} \beta_{d}^{2}}{\beta_{1}^{2}}-\frac{1}{r}\left[1+\frac{(r-1)(7 r-1) \beta_{d}^{2}}{3 \beta_{1}^{2}}\right]\left[1+\frac{2(3 r+2)(r-1) \beta_{d}^{2}}{5 \beta_{1}^{2}}\right]\right),
\end{gathered}
$$

for symmetric $\left(D_{\mu \mu}\left(-\mu_{1}\right)=D_{\mu \mu}\left(\mu_{1}\right)\right)$ upstream Fokker-Planck coefficients. Because the convection rate is always positive, cosmic-ray particles only are accelerated for a positive acceleration rate leading to the condition for the cosmic-ray velocities

$\beta_{1}>\beta_{c}(r)=\frac{\beta_{u} \sqrt{u_{c}(r)}}{r}, u_{c}(r)=\frac{45 r^{3}+(r-1)(53 r+7)}{30(r-1)}\left[1+\sqrt{\left.1+\frac{120(r-1)^{3}(3 r+2)(7 r-1)}{\left[45 r^{3}+(r-1)(53 r+7)\right]^{2}}\right]}\right.$,

which settles the injection threshold for nonrelativistic shock acceleration. Only cosmic-ray particles with momenta or velocities $y_{1} \simeq \beta_{1}>\beta_{c} \simeq y_{c}$ are accelerated by nonrelativistic shocks. The injection threshold velocity $\beta_{c}(r)$, shown in Fig. 1, for all flow compression ratios is always larger than $\sqrt{3} \beta_{u}$. 


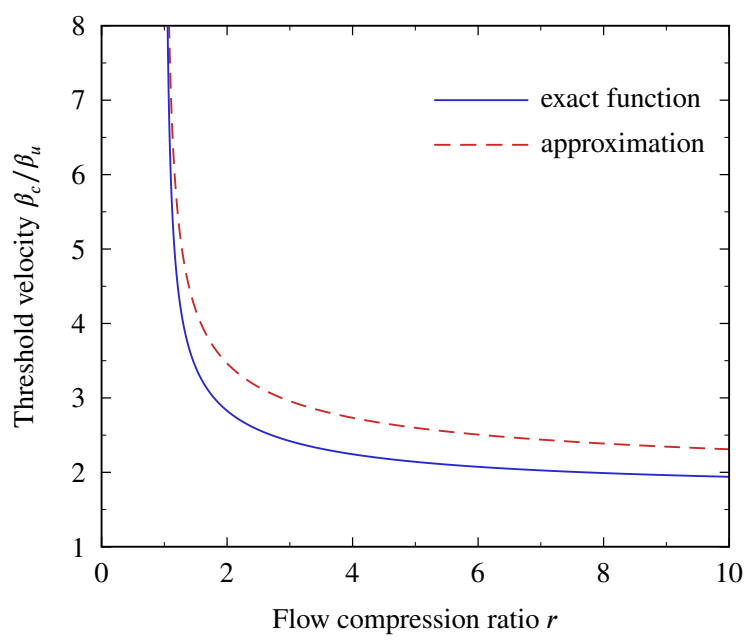

Figure 1: Injection threshold velocity $\beta_{c} / \beta_{u}$ as a function of the flow compression ratio $r$ for a nonrelativistic shock.

\subsection{Power-law spectral index}

With the rates (3.9) the power-law spectral index (3.7) is given by

$$
q\left(r, y_{1} \geq y_{0}>y_{c}\right)=3+\frac{2 y_{c}^{2} \beta_{1}^{2}}{y_{1}^{2}\left(\beta_{1}^{2}-y_{c}^{2}\right)}+\frac{3 r}{r-1} \frac{\beta_{1}^{2}-\frac{r-1}{3 r} \beta_{1}^{4}+\frac{r(r-1)^{2}(7 r-1) y_{c}^{2}}{9\left(r^{3}-r^{2}+1\right)}}{\beta_{1}^{2}-y_{c}^{2}},
$$

which is shown in Fig. 2 for $\beta_{1}>1.1 y_{c}$ and different flow compression ratios.

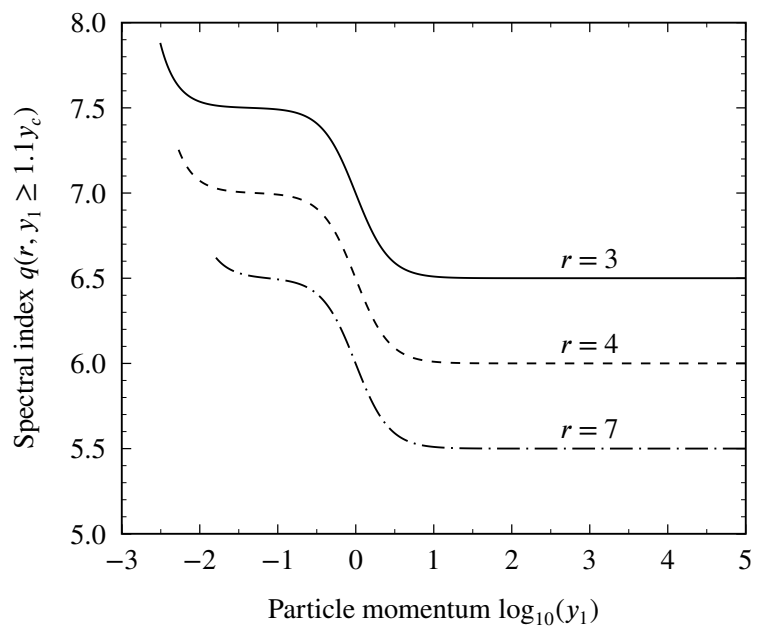

Figure 2: Power-law spectral index for a nonrelativistic shock with $\beta_{d}=10^{-4}$ as a function of particle momentum $y_{1}=1.1 y_{c}(r)$ for three different flow compression ratios $r=3,4,7$ (full curve).

For relativistic cosmic rays with $\beta_{1} \simeq 1 \gg y_{c}$ the spectral index (3.11) approaches $q\left(r, y_{1}>\right.$ $1)=q(r) \simeq(5 r-2 /(r-1)=2+(3 r /(r-1))$, which differs from the classical nonrelativistic shock acceleration theory spectral index $3 r /(r-1)$ by the additional factor 2 . 
For nonrelativistic cosmic rays with $\beta_{1} \simeq y_{1} \gg y_{c}$ the spectral index (3.11) approaches $q\left(r, y_{c} \ll\right.$ $\left.y_{1}<1\right)=q_{0}(r) \simeq=[3(2 r-1) /(r-1)]=1+q(r)$, which differs from the classical nonrelativistic shock acceleration theory spectral index $3 r /(r-1)$ by the additional factor 3 .

Obviously, our strictly relativistic shock acceleration theory, accounting correctly for the different momentum coordinates in the up- and downstream media, where the diffusion approximation has been applied, implies a much weaker efficiency for the acceleration of cosmic rays at nonrelativistic shocks, when the nonrelativistic limit of small but finite shock speeds in the relativistic theory is considered.

\section{Summary and conclusions}

The analytical theory of diffusive cosmic ray acceleration at parallel stationary shock waves of arbitrary speed has been developed. Starting from the Fokker-Planck particle transport equation in the mixed comoving coordinate system we derived for the first time the correct cosmic-ray jump conditions at the shock relating the upstream and downstream anisotropic cosmic-ray currents. The anisotropic upstream and downstream cosmic-ray currents are calculated from a diffusion approximation of particle transport in the upstream and downstream medium. Pitch-angle averaging the cosmic-ray current jump condition provides a general solution for the isotropic momentum spectrum of accelerated particles at the shock valid for arbitrary shock speeds, arbitrary cosmic-ray momenta and general injection functions at the shock, determined by the ratio of general acceleration and convection rates. The correctly taken limit for nonrelativistic shock speeds leads at relativistic cosmic-ray momenta to the power-law momentum spectrum $F_{1}\left(y_{1}\right) \propto y_{1}^{-q(r)}$ and at nonrelativistic cosmic-ray momenta to the power-law momentum spectrum $F_{1}\left(y_{1}\right) \propto y_{1}^{-(1+q(r))}$, where the power-law spectral index $q(r)$ is a factor 2 greater than the standard spectral index from nonrelativistic shock acceleration theory. Moreover, for nonrelativistic shock speeds we calculate for the first time the injection velocity threshold $\beta_{c} \geq \sqrt{3} \beta_{u}$, settling the long-standing injection problem for nonrelativistic shock acceleration.

\section{References}

[1] Gleeson, L. J., Axford, W. I., 1967, ApJ 149, L115

[2] Jokipii, J. R., 1976, ApJ 146, 480

[3] Kirk, J. G., Schneider, P., 1987, ApJ 315, 425

[4] Kirk, J. G., Schneider, P., Schlickeiser, 1988, ApJ 328, 269

[5] Lindquist, R. W., 1966, Ann. Phys. 37, 487

[6] Schlickeiser, R., Oppotsch, J., 2017, ApJ, to be submitted 